\title{
Acoustic radiation force due to a diverging wave: Demonstration and theory
}

\author{
Bruce C. Denardo, a) Stanley G. Freemyers, Michael P. Schock, and Scott T. Sundem \\ Department of Physics, Naval Postgraduate School, Monterey, California 93943
}

(Received 18 December 2012; accepted 30 October 2013)

\begin{abstract}
A radiation force is the time-averaged force exerted by any kind of wave on a body. In the case of a divergent traveling acoustic wave, it is known that a relatively small rigid body can experience a radiation force that is directed toward the source. We show that this effect can be readily demonstrated with a styrofoam sphere pendulum near a horizontally directed loudspeaker that is emitting sound of sufficiently high amplitude and low frequency. The attraction is surprising because repulsive forces are exerted by a traveling plane wave and by an outward jetting or "wind" from the loudspeaker. We argue that the attractive force near a source that is small compared to the wavelength can be roughly understood and calculated as a time-averaged Bernoulli effect, if scattering is ignored. The result is within a factor of two of rigorous published results based on scattering calculations, when these results are specialized to the case of a rigid body whose average density is much greater than the density of the fluid. However, repulsion occurs when the average density of the body is less than the density of the fluid, in which case our Bernoulli result completely fails. @ 2014 American Association of Physics Teachers.

[http://dx.doi.org/10.1119/1.4830097]
\end{abstract}

\section{INTRODUCTION}

The time-averaged force exerted by a wave on a body is called a radiation force. An example in acoustics is a traveling plane wave of wavelength $\lambda$ that is incident upon a rigid sphere of radius $a$. Consider first the geometrical (ray) acoustics limit in which the wavelength is small compared to the radius of the sphere $(\lambda / a \rightarrow 0)$, and when the sphere is relatively heavy so that its oscillations due to the acoustic field are negligible. In this case, the wave is strongly and symmetrically backward-scattered by the sphere. But a traveling sound wave carries momentum, ${ }^{1}$ which can be demonstrated in a high-intensity noise field by the attraction of two parallel plates $^{2}$ and by the rotation of an acoustic radiometer. ${ }^{3}$ By Newton's 2nd law, the change in momentum of the wave implies that the sphere exerts a backward force on the wave. By Newton's 3rd law, the wave must then exert a forward force on the sphere. It is reasonable, then, and has been calculated in a classic article, ${ }^{4}$ that the force exists in this direction for all values of the radius and mass of the sphere.

In the opposite limit of long wavelength compared to the radius of a sphere $(\lambda / a \rightarrow \infty)$, the radiation force due to a traveling plane wave vanishes because the amplitude of the scattered field vanishes in this limit. However, a common situation for relatively long wavelengths is that the wave is diverging rather than being strictly planar. In actual situations with sources of finite size in an unbounded or approximately unbounded medium, traveling plane waves typically occur only at sufficiently large distances from a source. It is thus surprising, and not well known, that the divergence of a traveling sound wave can cause a radiation force that is directed toward the source. This behavior was calculated ${ }^{5}$ and measured ${ }^{6}$ by Embleton for outgoing spherical waves incident upon a small rigid sphere, and has since been calculated for more general situations and other nonuniform waves by many investigators (see, for example, Refs. 7-13). In addition, there is current interest in possibly utilizing the effect for acoustic tweezers (see below) with a single traveling wave beam. ${ }^{14-17}$ For a divergent traveling wave, the force becomes repulsive at large distances due to the planar limit, but Embleton ${ }^{5}$ found that the attractive force due to a spherical wave can be much greater than the repulsive force even if the latter's inverse-square dependence with distance is taken into account.

The purpose of this article is to present a description and explanation of a simple classroom demonstration of the acoustic radiation force due to a diverging wave. Such a demonstration is not only interesting but is important due to the many applications of acoustic radiation forces. We focus on the radiation force of traveling waves, but a substantial amount of research has been devoted to standing waves as a result of the use of resonators for acoustic agglomeration (or coalescence) and levitation. ${ }^{18}$ Both of these effects can be demonstrated in a Kundt's tube with styrofoam chips. ${ }^{19}$ Applications include experiments in liquid drop oscillations on the Space Shuttle, ${ }^{20}$ single-bubble sonoluminescence, ${ }^{21}$ separation of oil droplets from water, ${ }^{22}$ and manipulation of biological cells. ${ }^{23}$ Another application is acoustic tweezers,${ }^{24}$ which conventionally consist of two oppositely directed traveling wave beams that produce a standing wave that can be used to manipulate small objects. Acoustic tweezers have recently been extended to surface acoustic waves. ${ }^{25}$ Some of the standing wave applications are also possible for divergent and convergent traveling waves. Indeed, one of the motivations for our research is the possible use of divergent waves to agglomerate unwanted particles in a liquid, so that the particles can then be removed.

Our demonstration is described in Sec. II, and a discussion and demonstration of the outward jetting from a loudspeaker is presented in Sec. III. A simple Bernoulli theory is developed in Sec. IV and is compared to the results of published scattering calculations in Sec. V. The theory is roughly correct for the demonstration, but completely fails for bodies whose average density is less than the density of the fluid. We also show in Sec. V that the attractive force of a significantly diverging wave is very much greater than the repulsive force due to a traveling plane wave of corresponding amplitude. Concluding remarks are made in Sec. VI. 


\section{DEMONSTRATION}

A classroom demonstration of the attractive radiation force due a diverging acoustic wave can be readily achieved with a loudspeaker near a pendulum consisting of a styrofoam sphere suspended by thread (Fig. 1). A ping-pong ball can alternatively be used, although greater acoustic amplitudes are required. We use a 6-in. diameter loudspeaker ${ }^{26}$ in an unported enclosure. The nominal impedance and maximum power are $8 \Omega$ and $150 \mathrm{~W}$, respectively, which correspond to a maximum drive amplitude of $35 \mathrm{~V}_{\mathrm{rms}}$. A small or low-quality loudspeaker may not deliver a sufficient acoustic amplitude for the effect to be observed. We usually support the loudspeaker with a lab jack in order to reduce any effect of acoustic reflections from the table. A function generator and power amplifier drive the loudspeaker at a frequency of $100 \mathrm{~Hz}$ (wavelength $3.4 \mathrm{~m}$ in air at room temperature). The styrofoam sphere has diameter $1.5 \mathrm{in}$. and mass $0.78 \mathrm{~g}$, and the pendulum has length $1.00 \mathrm{~m}$. The sphere must be sufficiently near the loudspeaker for the force to be observable, which is consistent with the radiation force decreasing very rapidly with distance (Sec. V). We adjust the apparatus such that the center of the sphere is $10.0 \mathrm{~cm}$ along the axis from the loudspeaker's dome. The sound level in the demonstration must be loud but need not be painful to the ear.

We were concerned that the high-amplitude sound wave in the demonstration could somehow cause the sphere to become electrically charged, and that this was at least partially responsible for the observed attraction. If this is the case, and the humidity is not high, the discharge after the sound is turned off should be sufficiently slow that the charge is easily detectable. However, the sphere returns to vertical equilibrium, and testing with an uncharged dielectric rod shows no interaction with the sphere. We also preheated the sphere with a hair dryer to reduce any discharging due to

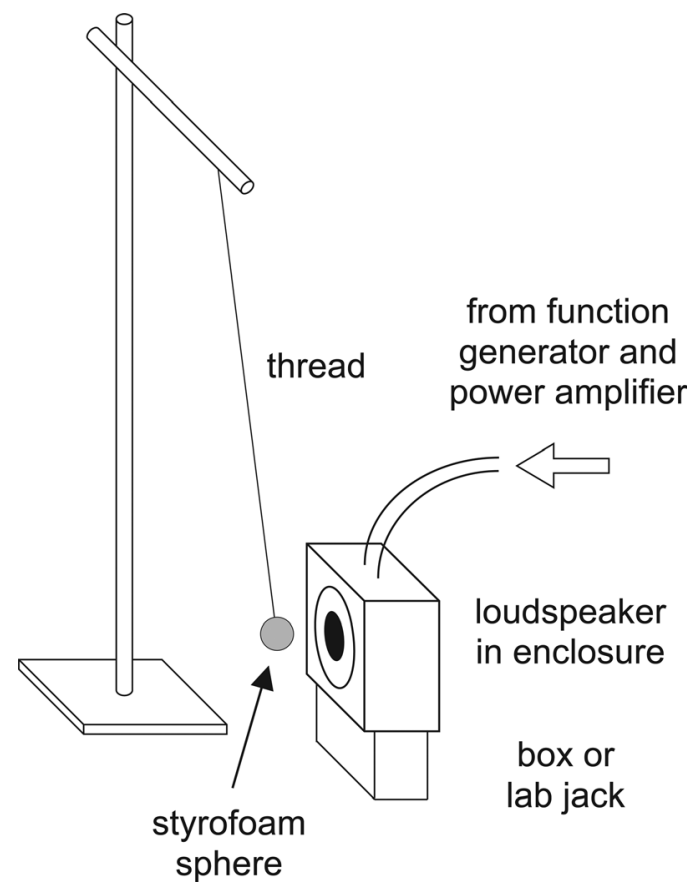

Fig. 1. Demonstration of the attractive acoustic radiation force due to a divergent traveling wave. The loudspeaker is driven at low frequency $(100 \mathrm{~Hz}$ is suitable) and high amplitude, which cause a visible attraction of a nearby lightweight body. water molecules, but we again observed no electrostatic interaction.

As the voltage across the loudspeaker is slowly increased from zero, no displacement of the sphere is observed at first. Only for a drive amplitude of about $15 \mathrm{~V}_{\mathrm{rms}}$ is it clearly apparent that the sphere has moved 3-4 mm toward the loudspeaker. The sound pressure level at a distance of $1.0 \mathrm{~m}$ on the axis of the loudspeaker is $93 \mathrm{~dB}$ ref $20 \mu \mathrm{Pa}$. A continued slow increase of the voltage causes the sphere to move more rapidly toward the loudspeaker. This behavior is a classic indication of a nonlinear acoustics effect that is proportional to the square of the amplitude of the sound field (Secs. III and IV). The displacement of the sphere from the vertical is clearly observable in a large classroom. At about $28 \mathrm{~V}_{\mathrm{rms}}$, when the center of the sphere is approximately $7.5 \mathrm{~cm}$ from the dome (a deflection of $2.5 \mathrm{~cm}$ ), an instability occurs in which the sphere moves relatively quickly into the loudspeaker, hits the dome, and is knocked outward. The sound pressure level is $97 \mathrm{~dB}$ at $1.0 \mathrm{~m}$ from the loudspeaker. The instability is explained below. The subsequent motion in which the sphere is continually knocked far outward by the loudspeaker is dramatic, and is essentially that of a driven bouncing ball, which is known to exhibit chaos. ${ }^{27}$

The instability can be understood as follows. For small horizontal displacements $x$ of the sphere from vertical equilibrium, the horizontal component of the tension in the thread increases linearly with $x$, while the acoustic radiation force has positive curvature. Furthermore, the radiation force increases with voltage amplitude $V$ across the loudspeaker. Magnitudes of the forces are shown qualitatively in Fig. 2, where $d$ is the distance between the sphere and the loudspeaker. For fixed $V$, equilibrium corresponds to a balance of the tension and the radiation force, and thus to intersections of the tension line and a radiation force curve. The graph shows that there is a critical value $V_{c}$ of the voltage. For $V<V_{c}$, there are two solutions, but only the smaller displacement is stable because an antirestoring force is seen to occur in a neighborhood of the larger displacement. For $V>V_{c}$, there are no solutions (the sphere hits the loudspeaker). For $V=V_{c}$, which corresponds to the observed instability, the

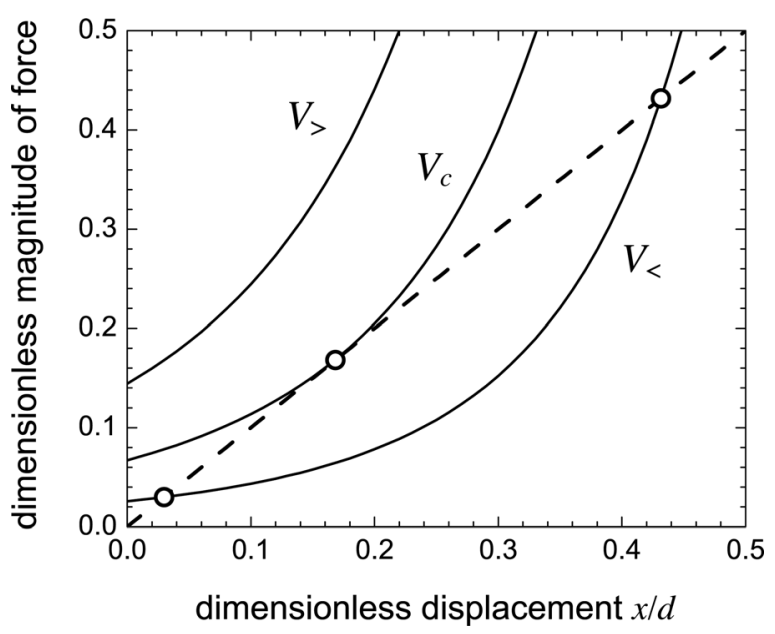

Fig. 2. Qualitative graph of the magnitudes of the acoustic radiation force (solid curves) and horizontal component of the tension (dashed line) for small displacements $x$ of a pendulum from vertical equilibrium. The loudspeaker is located at $x=d$. The curves correspond to different values of the voltage amplitude $V$ across the loudspeaker. Equilibrium occurs for the intersections (circles) of the curves and the line. 
equilibrium is unstable for positive fluctuations of $x$. The curves in Fig. 2 are qualitatively representative of the general case. They were quantitatively graphed for the simplest case of a spherical wave (Sec. IV) where the amplitude $A$ is taken to be proportional to the voltage $V$. The radiation force (Sec. V) is then $F_{\text {rad }}=V^{2} /(1-x / d)^{5}$ in dimensionless units, where $x=d$ corresponds to the center of the spherical wave. The magnitude of the dimensionless horizontal component of the tension is chosen to be $x / d$. The dimensionless voltages in Fig. 2 are $V_{<}=0.160, V_{c}=0.259$, and $V_{>}=0.380$.

We can easily calculate a typical value of the acoustic radiation force in the demonstration. For a relatively small equilibrium displacement $x$ of a simple pendulum of length $L$ and mass $m$, where the gravitational field strength is $g$, the magnitude of the acoustic radiation force is $F_{\text {rad }}=k x$, where the effective spring constant of the pendulum is $k=m g / L$. The mass-equivalent value of this force is then $m_{\text {rad }}$ $=F_{\text {rad }} / g=m x / L$. For our parameters $L=1.0 \mathrm{~m}$ and $m=0.78 \mathrm{~g}$, and the observed deflection $x=2.5 \mathrm{~cm}$ corresponding to the instability, we find $m_{\text {rad }}=20 \mathrm{mg}$, which is small by typical mechanical standards.

The demonstration exhibits attraction. Before the demonstration is observed, however, it is natural to guess that the sphere will either not move or will be repelled. No motion may be expected because the acoustic pressure rapidly oscillates in time, and the average force may be incorrectly thought to vanish. On the other hand, repulsion is expected for two distinct reasons. The first is the tendency due to the traveling plane wave radiation force (Sec. I). The second is jetting, which we discuss in Sec. III.

\section{LOUDSPEAKER JETTING}

At sufficiently high amplitudes, an outward jetting or "wind" can be produced by a loudspeaker. ${ }^{28}$ This jetting occurs due to a fundamental flow asymmetry in fluid mechanics. When an incompressible fluid is forced through an orifice and into the same fluid, the outflow tends to form a jet due to the separation of the boundary layer from the surface of the orifice; when the fluid is drawn in, the inflow is roughly isotropic. ${ }^{29}$ Symmetric flow only occurs for a sufficiently small Reynolds number that is much less than typical cases. A simple and dramatic example is the extinction of a candle flame by mouth, which can be readily accomplished by blowing but not by drawing in. A loudspeaker alternately blows and draws in, so the asymmetry yields a steady circulatory flow that is outward in the vicinity of the axis of the loudspeaker. A significant jet is expected to occur if the velocity amplitude of the loudspeaker is sufficiently large. This explanation of jetting assumes incompressible flow, which would appear to be violated in the case of acoustics. However, we can apply incompressible fluid dynamics in the case of the demonstration because the kinetic energy density is much larger than the potential energy density near an acoustic source that is small compared to the wavelength (Sec. IV).

The existence of jetting from the loudspeaker can be demonstrated as follows. The sphere is removed and the flame of a household butane lighter is held at the original equilibrium location of the center of the sphere, which is $10.0 \mathrm{~cm}$ on the axis from the dome of the loudspeaker. At $15 \mathrm{~V}_{\mathrm{rms}}$, for which the sphere moves $3-4 \mathrm{~mm}$ (see above), there is little or no bending of the flame. However, as the voltage is increased, there is an outward bending of roughly $45^{\circ}$ at the maximum voltage of $35 \mathrm{~V}_{\text {rms. }}$. This behavior is consistent with recently-published observations. ${ }^{30}$ At $7.5 \mathrm{~cm}$ and $28 \mathrm{~V}_{\text {rms }}$, for which the inward instability of the sphere occurs, a flame is extinguished horizontally outward. The effect here is different from the demonstration ${ }^{30}$ of a flame in a very-low-frequency sound field (roughly $3 \mathrm{~Hz}$ ), where the flame responds at the frequency of the sound. This response is negligible for our case of $100 \mathrm{~Hz}$, but there does occur a steady outward deflection due to the time-averaged flow.

The above explanation of loudspeaker jetting due to the flow asymmetry may not be complete. The flow due to an orifice in a fluid that is acoustically driven at high amplitudes can exhibit very complicated behavior, as observed by Ingard and Labate. ${ }^{31}$ The behavior includes vortex ring shedding at the frequency of the drive and even an inward flow along the axis in some cases. However, a loudspeaker in an unported enclosure is only similar to an orifice, so the existence of such flows is not clear. A search of the literature did not yield any considerations of these possibilities. The search did reveal a substantial current interest in synthetic jets, which are net-zero-mass flows that can occur due to Helmholtz resonators ${ }^{32}$ with volume-modulated cavities. These systems may be employed as active control devices in a variety of fluid flow applications. ${ }^{33}$ Synthetic jet researchers typically define a jet as a flow that results from vortex ring shedding, which indicates that the outward flow due to just the asymmetry is much weaker. However, the shedding only occurs if the particle velocity amplitude in an orifice exceeds a threshold value, ${ }^{34}$ which may not be readily attainable for loudspeakers. To the best of our knowledge, further research on the jetting from loudspeakers needs to be done.

\section{BERNOULLI THEORY}

In the demonstration shown in Fig. 1, what is breaking the symmetry of the zero-time-average of the linear pressure oscillations and overcoming the repulsive effects of both plane-wave scattering and jetting? The answer is the sound wave's nonuniformity, which in this case is the divergence. The simplest type of diverging acoustic wave is a spherical wave, which is isotropic. The linear acoustic pressure $p$ in general satisfies the standard wave equation, which has the monofrequency outgoing spherical wave solution ${ }^{32}$

$$
p=\frac{A}{r} \cos (\omega t-k r),
$$

where $A$ is a constant, $r$ is the distance from the center of the source, $\omega$ is the angular frequency, $t$ is the time, and $k$ is the wave number. The frequency and wave number are related as $\omega=c k$, where $c$ is the speed of sound.

The particle velocity $\mathbf{u}$ of an outgoing spherical wave can be determined from the linearized Euler's equation ${ }^{1}$ (Newton's second law for a fluid element)

$$
\rho_{0} \frac{\partial \mathbf{u}}{\partial t}=-\nabla p,
$$

where $\rho_{0}$ is the equilibrium density of the fluid. For purely radial motion $\mathbf{u}=u(r, t) \hat{r}$ and Eq. (2) reduces to $\rho_{0} \partial u / \partial t$ $=-\partial p / \partial r$. Substituting Eq. (1) into the right-hand-side, integrating with respect to time, and simplifying, yields 


$$
u=\frac{A}{\rho_{0} c r}\left[\cos (\omega t-k r)+\frac{1}{k r} \sin (\omega t-k r)\right] .
$$

In contrast to the pressure in Eq. (1), the particle velocity is not simply proportional to $1 / r$ unless $k r \gg 1$ (the plane wave limit). In what follows we use the spherical wave pressure solution (1) and corresponding velocity solution (3) to motivate and understand a general expression for the acoustic radiation force on a body.

In general, the instantaneous acoustic potential and kinetic energy densities of a sound wave are ${ }^{32}$

$$
e_{p}=\frac{1}{2 \rho_{0} c^{2}} p^{2} \quad \text { and } \quad e_{k}=\frac{1}{2} \rho_{0} u^{2},
$$

which are valid to leading (second) order in the acoustic variables. For $k r \gg 1$, an outgoing spherical wave is locally a traveling plane wave. In this case, Eqs. (1), (3), and (4) yield the equipartition of energy $e_{k}=e_{p}$. At the other extreme, however, $k r \ll 1$ yields $e_{k} \gg e_{p}$. This situation occurs near a "small" source, where the size of the source is small compared to the wavelength. The large imbalance in the kinetic and potential energy densities is due to the sine term in Eq. (3). Physically, the strong divergence of the motion causes substantial circumferential compressions and expansions which correspond to a nearly $90^{\circ}$ phase difference of pressure and particle velocity. ${ }^{32}$ Energy flows back and forth between the source and near field, with only a relatively small amount being radiated, which explains why a small acoustic source is an inefficient radiator.

Because the kinetic energy dominates the potential energy near a small source, the flow is approximately and surprisingly incompressible in this region, so the well-known standard Bernoulli relationship between pressure and velocity applies. The time average of this relationship yields

$$
p_{\text {rad }}+\frac{1}{2} \rho_{0}\left\langle u^{2}\right\rangle=\text { const }=0,
$$

where the brackets denote time averaging, and where $p_{\text {rad }}=\langle p\rangle$ is the acoustic radiation pressure. The value of the constant in Eq. (5) is zero in our case because the diverging nature of the sound wave causes the field to approach zero as the distance from the source increases, and so the radiation pressure must vanish in this limit. From the kinetic energy density in Eq. (4), the radiation pressure in Eq. (5) is $p_{\text {rad }}=-\left\langle e_{k}\right\rangle$ for our case of approximately incompressible flow. For a body that is small compared to the wavelength the scattering is small, so it would appear that the particle velocity in Eq. (5) can be determined from the acoustic field in the absence of the body (however, see Sec. V). The radiation force is then determined from the radiation pressure in the same way that the force on a small element of fluid is determined from the pressure in the derivation of Euler's equation. ${ }^{1}$ Specifically, the force per unit volume is the negative of the gradient of the pressure:

$$
\frac{\mathbf{F}_{\mathrm{rad}}}{V}=-\nabla p_{\mathrm{rad}}=\nabla\left\langle e_{k}\right\rangle,
$$

where $V$ is the volume of the body and $e_{k}$ is the incident kinetic energy density.

A simple but naive (see Sec. V) explanation of the demonstration shown in Fig. 1 is then as follows. The particle velocity decreases with distance from the source, so the radiation pressure increases according to the Bernoulli relationship in Eq. (5). This imbalance in radiation pressure yields an attractive force on a body due to the acoustic field, as observed in the demonstration. Furthermore, the radiation pressure is nonlinear due to the square of the particle velocity $u$, which is consistent with the behavior in the demonstration. Note that the argument leading to the Bernoulli force in Eq. (6) also applies to a converging wave with $k r \ll 1$, so there will again be a force toward the center of the wave.

Although we have considered an outgoing spherical wave in order to motivate and understand the approximation near a small source $(k r \ll 1)$ in the derivation of the Bernoulli relationships [Eqs. (5) and (6)], these relationships are expected to hold in general when the typical local radius of curvature of a wave front is small compared to the wavelength, so that the kinetic energy density dominates the potential energy density. Indeed, the radiation pressure to second order for any acoustic field can be rigorously shown ${ }^{1}$ to be $p_{\text {rad }}=\left\langle e_{p}\right\rangle-\left\langle e_{k}\right\rangle+$ constant. The Bernoulli relationships correspond to the special case $e_{p} \ll e_{k}$ that occurs near a small source.

\section{RESULTS OF SCATTERING CALCULATIONS}

In this section, we show that there is a fundamental mistake in the Bernoulli theory (Sec. IV) and we discuss the correct result. We also show that the acoustic radiation force due to a significantly divergent wave has a much greater magnitude than that due to a traveling plane wave of corresponding amplitude.

Because the wavelength is assumed to be much greater than the size of the body the scattered field is small, and neglecting it as in Sec. IV might appear to be a good approximation. However, what is relevant for the force on the body is the field at the surface of the body, and this field is substantially influenced by scattering. A correct approach to determine the second-order radiation force on a body is to time average the integral of the force due to the total pressure at each point of the surface of the body. This pressure consists of the total linear pressure (incident plus scattered), which contributes due to oscillations of the body, ${ }^{4,9}$ and the second-order radiation pressure $p_{\text {rad }}=\left\langle e_{p}\right\rangle-\left\langle e_{k}\right\rangle$ (Sec. IV), where the energy densities here correspond to the total field (incident plus scattered).

Utilizing King's work, ${ }^{4}$ Nyborg $^{9}$ has performed this calculation for a rigid sphere on the axis of a general axisymmetric sound field, where viscous and thermal effects are ignored. The radius of the sphere is assumed to be small compared to the characteristic length of variation of the sound field. The use of a sphere is for mathematical convenience only; the same results are expected to hold for any small rigid body that is free to oscillate due to the acoustic field. Specializing Nyborg's result to the case of an incident sound wave that is sufficiently different from a traveling plane wave yields the following radiation force per unit volume of the body:

$$
\frac{F_{\mathrm{rad}}}{V}=B \frac{d}{d z}\left\langle e_{k}\right\rangle-\frac{d}{d z}\left\langle e_{p}\right\rangle
$$

where $z$ is the distance along the axis of symmetry, and $e_{k}$ and $e_{p}$ are the local incident sound energy densities (in the absence of the sphere). The coefficient of the kinetic energy 
density term is $B=3\left(\rho_{b}-\rho_{0}\right) /\left(2 \rho_{b}+\rho_{0}\right)$, where $\rho_{0}$ is the equilibrium density of fluid (as in Sec. IV) and $\rho_{b}$ is the average density of the body. Figure 3 shows a graph of $B$ as a function of the ratio of the densities $\rho_{0} / \rho_{b}$. The common case of a relatively heavy body $\left(\rho_{b} \gg \rho_{0}\right)$ yields $B=3 / 2$. In addition, near a small source $(k r \ll 1)$, the potential energy term in Eq. (7) is negligible (Sec. IV), so this equation yields an attractive force that differs by a factor of $3 / 2$ from the simple Bernoulli result [Eq. (6)] that ignores scattering.

The radiation force in Eq. (7) depends upon the mass of the body because the acoustic field causes the body to oscillate, which affects the scattering such that the force is reduced relative to the value for a heavy body $\left(\rho_{b} \gg \rho_{0}\right)$. The oscillations evidently increase the backward scattering relative to the forward scattering. The reduction in force increases for lighter bodies, and can eventually cause the force to be repulsive rather than attractive. Indeed, near a small source the potential energy term in Eq. (7) is negligible, and a body with density $\rho_{b}<\rho_{0}$ has $B<0$ (Fig. 3), so the radiation force is repulsive. The Bernoulli theory is then completely inaccurate in this case.

In regard to the demonstration shown in Fig. 1, the density of the styrofoam sphere is calculated to be $\rho_{b}=27 \mathrm{~kg} / \mathrm{m}^{3}$, while the density of air at room temperature and atmospheric pressure is $\rho_{0}=1.2 \mathrm{~kg} / \mathrm{m}^{3}$. The value of the kinetic energy density coefficient in Eq. (7) is then $B=1.4$, which is near the value 1.5 for the limiting case of a heavy body.

As explained above, the potential energy term in the acoustic radiation force (7) is negligible near a small source, so the flow is approximately incompressible. The kinetic energy density term in Eq. (7) must then be identical to the force for incompressible flow. Specifically, the coefficient $B$ must arise from the fact ${ }^{1,35}$ that the velocity $v$ of a sphere due to an oscillating inviscid incompressible fluid of velocity $u$ is $v=3 \rho_{0} u /\left(2 \rho_{b}+\rho_{0}\right)$. Furthermore, the attraction for $\rho_{b}>\rho_{0}$ and the repulsion for $\rho_{b}<\rho_{0}$ must be understandable from this result, which may offer a more physical interpretation than the acoustical scattering theory. We leave these considerations for possible future work.

The radiation force per unit volume given in Eq. (7) is remarkably general, holding for incident divergent and convergent traveling waves, planar and nonplanar standing

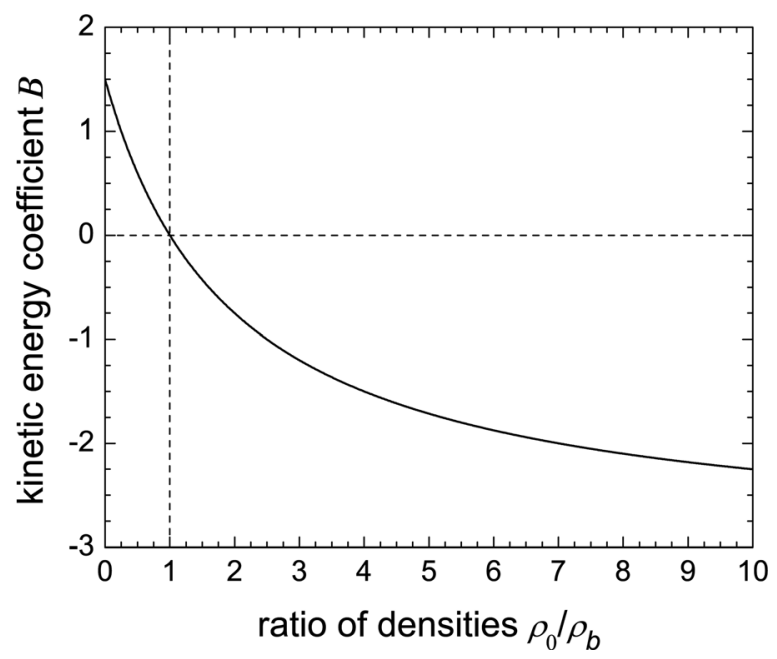

Fig. 3. Nyborg's dimensionless kinetic energy density coefficient $B$ as a function of the ratio of the fluid density $\rho_{0}$ to the average body density $\rho_{b}$. The asymptotic value as $\rho_{0} / \rho_{b} \rightarrow \infty$ is $B=-3$. waves, and superpositions of these waves, although it does not hold for traveling plane waves. An explicit expression for the special case of a traveling spherical wave (Sec. III) can be easily obtained. We again assume that the body is near a small source $(k r \ll 1)$. Substitution of Eq. (1), (3), and (4) into Eq. (7) yields the following radiation force per unit volume to leading order in $k r$, which is the inverse 5 th power, or

$$
\frac{F_{s p h}}{V}=-B \frac{A^{2}}{\rho_{0} \omega^{2} r^{5}} .
$$

The magnitude of the radiation force decreases rapidly with increasing frequency $\omega$. In addition, the magnitude decreases very rapidly with increasing distance $r$, which is consistent with the fact that the sphere in the demonstration must be sufficiently near the loudspeaker for the effect to be observed, and that the force is sensitive to changes in the equilibrium distance. That the effect is nonlinear, which is also indicated in the demonstration, is evidenced by the force being proportional to the square of the constant $A$ in Eq. (8), while the acoustic pressure (1) and particle velocity (3) are both proportional to $A$.

In addition to the radiation force [Eqs. (7) or (8)] on a body, there is a tendency for repulsion due to the plane wave effect (Sec. I). However, we can show that this force is negligible for a small sphere of radius $a$ near a small source $(k a \ll k r \ll 1)$. We consider the standard case ${ }^{1}$ of the repulsive force exerted by a traveling plane wave on a fixed rigid sphere (corresponding to $\rho_{b} \gg \rho_{0}$ ), where the radius $a$ of the sphere is much less than the wavelength. The force is $F_{\mathrm{pl}}=11 \pi \omega^{4} a^{6}\langle e\rangle / 9 c^{4}$, where $\langle e\rangle$ is the time-averaged energy density of the incident wave. The volume is $V=4 \pi a^{3} / 3$, so the force per unit volume is $F_{\mathrm{pl}} / V=11 \omega^{4} a^{3}\langle e\rangle / 12 c^{4}$. The ratio of the plane wave radiation force $F_{\mathrm{pl}}$ to the spherical wave radiation force $F_{\mathrm{sph}}$ in Eq. (8) with $B=3 / 2$ then has magnitude

$$
\left|\frac{F_{\mathrm{pl}}}{F_{\mathrm{sph}}}\right|=\frac{11 \rho_{0} \omega^{6} a^{3} r^{5}\langle e\rangle}{18 c^{4} A^{2}},
$$

To analyze this ratio, we could consider equal pressure amplitudes of the spherical and planar waves at the location of the sphere. However, this comparison is an underestimate of the ratio because, from Eqs. (1) and (3), the particle velocity amplitude of a spherical wave near a small source is much greater than the particle velocity amplitude of a plane wave with the same pressure amplitude. We thus consider equal particle velocity amplitudes. From Eq. (3), the amplitude is $U=\left(A / \rho_{0} c r\right)\left[1+1 /(k r)^{2}\right]^{1 / 2} \approx A / \rho_{0} c k r^{2}$ for $k r \ll 1$. Substituting this expression into the traveling plane wave average energy density $\langle e\rangle=\rho_{0} U^{2} / 2$ (Sec. IV) and then substituting the resultant expression into the force ratio in Eq. (9) yields $\left|F_{\mathrm{pl}} / F_{\mathrm{sph}}\right|=(11 / 36)(k a)^{3}(k r)$. Because $k a \ll k r \ll 1$, we see that the plane wave force is very much smaller than the spherical wave force. Using a different approach based on his more-general solution, Nyborg ${ }^{9}$ arrives at the same dependence on $k a$ and $k r$.

\section{CONCLUDING REMARKS}

A common situation for traveling waves in an unbounded fluid is that they diverge from a source. In the case of 
acoustics, this divergence can cause an attractive timeaveraged force on a relatively small rigid body near the source. The force is an example of an acoustic radiation force, which has many useful applications. A simple classroom demonstration can readily be done with a styrofoam ball pendulum near a loudspeaker that is driven at sufficiently high amplitude and low frequency. The attraction occurs even though a plane wave exerts a repulsive force, and even though there is an outward jetting from the loudspeaker. Due to the low frequency, the wave that is emitted from the loudspeaker is highly divergent, but there is still a tendency for plane-wave repulsion. The jetting can be demonstrated with a flame that is held near the loudspeaker, and arises from a flow asymmetry in fluid mechanics, although additional effects may be present.

If scattering is ignored, the attractive force on the body can be roughly understood and calculated as a time-averaged Bernoulli effect, which is nonlinear. The flow is approximately incompressible near the source due to the high divergence of the sound wave. Scattering calculations in the literature show that the radiation force on a small rigid body arises from the nonuniformity of the incident wave in competition with the oscillatory motion of the body due to the wave. Near a source that is small compared to the wavelength, the force is attractive if the average density of the body is greater than the density of the fluid. The force is repulsive for the opposite case, which represents a complete failure of the Bernoulli theory. Scattering calculations also show that the attractive force due to a significantly divergent wave has a much greater amplitude than that due to the repulsive force of a traveling plane wave with the same particle velocity amplitude. The tendency for repulsion in this case is thus negligible.

In regard to a quantitative experimental verification of the theoretical acoustic radiation force in Eq. (7), Embleton performed a shielded pendulum experiment with outgoing spherical waves that resulted from piped-in sound. ${ }^{6}$ The shielding eliminated any force due to jetting, and the radiation force was measured by observing the deflection of the pendulum with a telescope. We have measured the force with a purely vertical arrangement of a loudspeaker, sphere, and analytical balance. In addition, the waves in our experiment are not spherical and the jetting is not shielded. The results will be reported in an article that is currently being prepared. $^{36}$

\section{ACKNOWLEDGMENTS}

The authors are very grateful for the assistance of summer intern Alissa Bell and students Joseph Beach, Rebecca King, Ana Vieira, and Steven Yang. The authors are also very grateful for discussions with Professor Steven Baker and Professor Andrés Larraza, and for suggestions by the journal's reviewers.

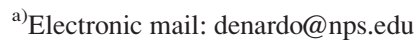

${ }^{1}$ L. D. Landau and E. M. Lifshitz, Fluid Mechanics, 2nd ed. (ButterworthHeinemann, New York, 1987), Secs. 2, 11 (Prob. 1), 64, 65, and 78 (Prob. 4).

${ }^{2}$ Andrés Larraza, "A demonstration apparatus for an acoustic analog to the Casimir effect,” Am. J. Phys. 67, 1028-1030 (1999).

${ }^{3}$ Bruce Denardo and Timothy G. Simmons, "An acoustic radiometer," Am. J. Phys. 72, 843-845 (2004).

${ }^{4}$ Louis V. King, "On the acoustic radiation pressure on spheres," Proc. R. Soc. London, Ser. A 147, 212-240 (1934).
}

${ }^{5}$ T. F. W. Embleton, "Mean Force on a sphere in a spherical sound field. I. (Theoretical)," J. Acoust. Soc. Am. 26, 40-45 (1954).

${ }^{6}$ T. F. W. Embleton, "Mean force on a sphere in a spherical sound field. II. (Experimental)," J. Acoust. Soc. Am. 26, 46-50 (1954).

${ }^{7}$ T. F. W. Embleton, "The radiation force on a spherical obstacle in a cylindrical sound field," Can. J. Phys. 34, 276-287 (1956).

${ }^{8}$ L. P. Gor'kov, "On the forces acting on a small particle in an acoustical field in an ideal fluid," Sov. Phys. Dokl. 6, 773-775 (1962).

${ }^{9}$ Wesley L. Nyborg, "Radiation pressure on a small rigid sphere," J. Acoust. Soc. Am. 42, 947-952 (1967).

${ }^{10}$ Takahi Hasegawa, Masayuki Ochi, and Kiichiro Matsuzawa, "Acoustic radiation force on a solid elastic sphere in a spherical wave field," J. Acoust. Soc. Am. 69, 937-942 (1981).

${ }^{11}$ Xucai Chen and Robert E. Apfel, "Radiation force on a spherical object in an axisymmetric wave field and its application to the calibration of highfrequency transducers," J. Acoust. Soc. Am. 99, 713-724 (1996).

${ }^{12}$ Alexander A. Doinikov, "Acoustic radiation force on a spherical particle in a viscous heat-conducting fluid. I. General formula," J. Acoust. Soc. Am. 101 (2), 713-721 (1997). In the same issue, see also the two succeeding articles by the same author: "II. Force on a rigid sphere," pp. 722-730, and "III. Force on a liquid drop," pp. 731-740.

${ }^{13}$ S. D. Danilov and M. A. Mironov, "Mean force on a small sphere in a sound field in a viscous fluid," J. Acoust. Soc. Am. 107, 143-153 (2000).

${ }^{14}$ Jungwoo Lee, Kanglyeol Ha, and K. Kirk Shung, "A theoretical study of the feasibility of acoustical tweezers: Ray acoustics approach," J. Acoust. Soc. Am. 117, 3273-3280 (2005).

${ }^{15} \mathrm{~F}$. G. Mitri, "Negative axial radiation force on a fluid and elastic spheres illuminated by a high-order Bessel beam of progressive waves," J. Phys. A: Math. Theor. 42, 245202-1-9 (2009).

${ }^{16}$ Glauber T. Silva, "An expression for the radiation force exerted by an acoustic beam with arbitrary wavefront," J. Acoust. Soc. Am. 130, 3541-3544 (2011).

${ }^{17}$ Likun Zhang and Philip L. Marston, "Axial radiation force exerted by general non-diffracting beams," J. Acoust. Soc. Am. 131, EL329-EL335 (2012).

${ }^{18}$ T. G. Wang and C. P. Lee, "Radiation Pressure and Acoustic Levitation," in Nonlinear Acoustics, edited by Mark F. Hamilton and David T. Blackstock (Academic, San Diego, 1998), Chap. 6.

${ }^{19}$ Lecture demonstrations manual, Department of Physics and Astronomy, University of California at Los Angeles, <http://www.physics.ucla.edu/ demoweb/demomanual/acoustics/effects_of_sound/kundts_tube.html $>$.

${ }^{20}$ C. P. Lee, A. V. Anilkumar, A. B. Hmelo, and T. G. Wang, "Equilibrium of liquid drops under the effects of rotation and acoustic flattening: Results from USML-2 experiments in Space," J. Fluid Mech. 354, 43-67 (1998). See also E. H. Trinh, R. G. Holt, and D. B. Thiessen, "The dynamics of ultrasonically levitated drops in an electric field," Phys. Fluids 8, 43-61 (1996).

${ }^{21}$ Seth J. Putterman, "Sonoluminescence: Sound into Light," Sci. Am. 272, 46-51 (February, 1995). In the same issue, see also Robert A. Hiller, and P. Barber, "Producing Light from a Bubble of Air," pp. 96-98.

${ }^{22}$ G. D. Pangu and D. L. Feke, "Kinetics of ultrasonically induced coalescence within oil/water emulsions: Modeling and experimental studies," Chem. Eng. Sci. 64, 1445-1454 (2009).

${ }^{23}$ W. T. Coakley, J. J. Hawkes, M. A. Sobanski, C. M. Cousins, and J. Spengler, "Analytical scale ultrasonic standing wave manipulation of cells and microparticles," Ultrasonics 38, 638-641 (2000). W. Terrence Coakley, David W. Bardsley, Martin A. Grundy, Freidoun Zamani, and David J. Clarke, "Cell manipulation in ultrasonic standing wave fields," J. Chem. Tech. Biotechnol. 44, 43-62 (1989).

${ }^{24}$ F. G. Mitri, "Acoustic radiation force of high-order Bessel beam standing wave tweezers on a rigid sphere," Ultrasonics 49, 794-798 (2009).

${ }^{25}$ Xiaoyun Ding, Sz-Chin Steven Lin, Brian Kiraly, Hongjun Yue, Sizing Li, I-Kao Chiang, Jinjie Shi, Stephen Benkovic, and Tony Jun Huang, "Onchip manipulation of single microparticles, cells, and organisms using surface acoustic waves," Proc. Natl. Acad. Sci. U.S.A. 109, 11105-11109 (2012).

${ }^{26} \mathrm{JL}$ Audio 6W3v3-8 loudspeaker (JL Audio, Miramar, Florida), <http:// www.jlaudio.com $>$. The dome is inverted in this case, but we have also successfully done the demonstration with normally protruding domes.

${ }^{27}$ Nicholas B. Tufillaro, Tyler Abbott, and Jeremiah Reilly, An Experimental Approach to Nonlinear Dynamics and Chaos (Addison-Wesley, Redwood City, California, 1992), Ch. 1.

${ }^{28}$ Jan Abildgaard Pedersen and John Vanderkooy, "Near-Field Acoustic Measurements at High Amplitudes," 104th Audio Engineering Society Convention, Amsterdam, Paper No. 4683 (May 1998). 
${ }^{29}$ T. E. Faber, Fluid Dynamics for Physicists (Cambridge U.P., New York, 1995), pp. 64-65, 263-264.

${ }^{30}$ Zdeslav Hrepic, Corey Nettles, and Chelsea Bonilla, "Demonstrating sound wave propagation with candle flame and loudspeaker," Phys. Teach. 51, 16-19 (2013)

${ }^{31} \mathrm{U}$. Ingard and S. Labate, "Acoustic circulation effects and the nonlinear impedance of orifices," J. Acoust. Soc. Am. 22, 211-218 (1950).

${ }^{32}$ Lawrence E. Kinsler, Austin R. Frey, Alan B. Coppens, and James V. Sanders, Fundamentals of Acoustics, 4th ed. (Wiley, New York, 2000), Secs. 5.8, 5.11, 7.1, and 10.8.
${ }^{33}$ Ari Glezer and Michael Amitay, "Synthetic jets," Annu. Rev. Fluid Mech. 34, 503-529 (2002).

${ }^{34}$ Ryan Holman, Yogen Utturkar, Rajat Mittal, Barton L. Smith, and Louis Cattafesta, "Formation criterion for synthetic jets," Am. Inst. Aero. Astro. (AIAA) J. 43, 2110-2116 (2005).

${ }^{35}$ Thomas B. Gabrielson, David L. Gardner, and Steven L. Garrett, "A simple neutrally buoyant sensor for direct measurement of particle velocity and intensity in water," J. Acoust. Soc. Am. 97, 2227-2237 (1995).

${ }^{36}$ Bruce C. Denardo, Justin E. Ivancic, Eric C. Oviatt, Konstantinos K. Patsiaouras, Steven H. Yang, and Mohamed Akram Zrafi, "Acoustic radiation force due to a diverging wave: Experiment," (in preparation)

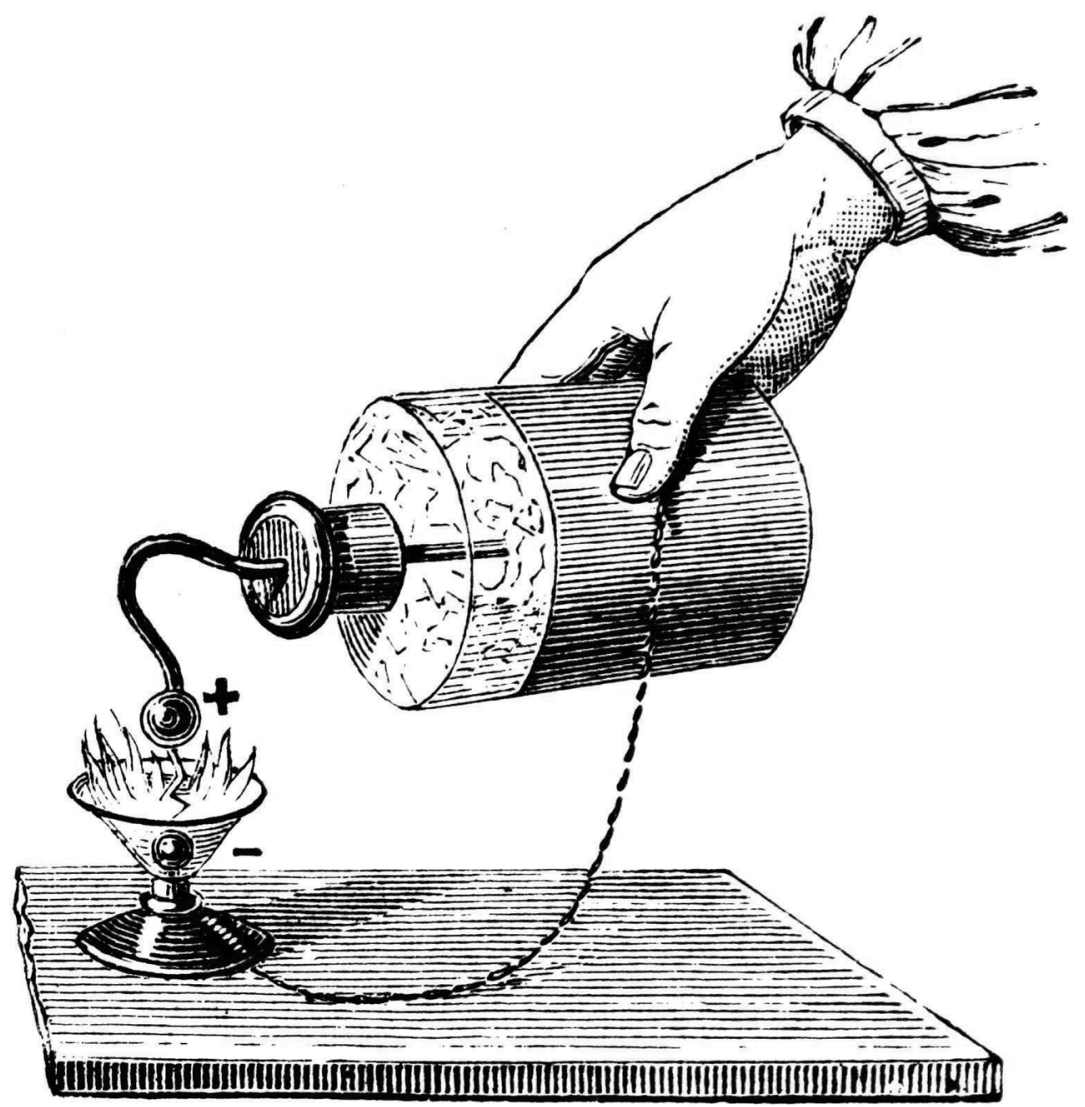

Setting Ether Alight

This may not be a demonstration that you wish to do today, although it was quite standard in the $19^{\text {th }}$ century lecture hall. Ether has a high enough vapor pressure to allow it to be easily ignited, in the presence of the oxygen of the air, with the spark from a Leiden jar. Ganot, whose book is as much a manual of demonstrations as a textbook, tells us that alcohol must be warmed to get enough vapor into the air to allow it to be lit. From A. Ganot, Elementary Treatise on Physics, trans. and ed. By E. Atkinson (Longmans, Green, and Co., London, 1883) pg 708. (Text by Thomas B. Greenslade, Jr., Kenyon College) 